\title{
Las prácticas comunicativas online y su impacto en las actitu- des políticas de las y los jóvenes en la zona Metropolitana de Monterrey Nuevo León
}

Online communication practices and their impact on the political activities of young people in the Metropolitan area of Monterrey Nuevo León

\author{
Juan de Dios Martínez Villarreal \\ Universidad Autónoma de Nuevo León \\ Orcid http://orcid.org/oooo-0oo2-5868-3786 \\ juandediosmtzv@hotmail.com
}

\author{
Pedro Paul Rivera Hernández \\ Universidad Autónoma de Nuevo León \\ Orcid https://orcid.org/oooo-0oo2-2137-2538 \\ pedro_riverahdz@hotmail.com
}

\author{
Devany Ana Karen Covarrubias Alemán \\ Universidad Autónoma de Nuevo León \\ Orcid https://orcid.org/oooo-0001-9405-1789 \\ devany.covarrubias@gmail.com
}

\author{
Fernando Girón Bárcenas \\ Universidad Autónoma de Nuevo León \\ Orcid https://orcid.org/o0oo-0002-7014-8444 \\ bunquer924@hotmail.com
}

\begin{abstract}
Resumen: El uso de internet simboliza una nueva práctica dentro de la comunicación política que tendrá un impacto en las actitudes políticas de las y los jóvenes. Éstos representan a una parte muy importante de la población en la Zona Metropolitana de Monterrey, Nuevo León México (ZMM); aproximadamente el 28.75\% (a marzo de 2019) de la lista nominal. La metodología que se utilizó fue de corte cuantitativo, ya que se aplicó encuesta de opinión a las y los jóvenes entre 18 y 29 años. Los objetivos planteados son los siguientes: identificar los niveles de uso de internet en materia política, de interés en la política, de eficacia política interna y de confianza en las instituciones políticas y de gobierno. Asimismo, se tiene la intención de determinar si el uso de internet en lo que corresponde al área política explica las diferentes actitudes políticas de los jóvenes en la ZMM. Los resultados más relevantes muestran que los niveles de interés en la política y la sensación de eficacia política interna son positivos. Por otra parte, la confianza en las instituciones políticas y de gobierno es muy baja, mientras que el menor nivel de confianza se tiene frente a los partidos políticos y la Presidencia de la República. Es importante resaltar que el uso de internet con fines políticos presenta un nivel explicativo relevante frente al interés en la política y medianamente importante sobre la sensación de eficacia política interna.
\end{abstract}

Palabras clave: Conversación política interactiva, consumo de contenidos políticos online, interés en la política, eficacia política, confianza en instituciones políticas y de gobierno 


\begin{abstract}
The use of Internet symbolizes a new practice within political communication that will have an impact on the political attitudes of young people; these represent a crucial part of the population in the Metropolitan Area of Monterrey Nuevo Léon México (ZMM) approximately 28.75\% (to March 2019) of the nominal list. The methodology used was quantitative because a poll of opinion was applied to young people between 18 and 29 years. The objectives are the following: to identify levels of internet use in political matters, interest in politics, internal political efficacy and, trust in political and government institutions. Also, it is intended to determine if the use of the internet in what corresponds to the political area explains the different political attitudes of young people in the ZMM. The most relevant results show that the levels of interest in politics and the sensation of internal political efficiency are positive, on the other hand, the trust in the political and government institutions is deficient, the lower trust is facing the parties politicians and the Presidency of the Republic. It is essential to highlight that the use of the internet for political purposes presents a relevant explanatory level to the interest in politics and moderately important in the sensation of internal political efficacy.
\end{abstract}

Keywords: Interactive political conversation, consumption of online political content, interest in politics, political efficiency, trust in political and government institutions

Fecha de recepción: 03/06/2019

Fecha de aprobación: 22/08/2019

Cómo citar este artículo / How to cite this paper: Martínez Villarreal, J. D., Rivera Hernández, P. P. Covarrubias Alemán, D. A. K., \& Girón Bárcenas, F. (2019). El uso político de internet y su impacto en las actitudes políticas de las y los jóvenes en la zona Metropolitana de Monterrey Nuevo León. Revista de Comunicación Política, 1, 31-52. doi:10.29105/rcp1-2 


\section{Introducción}

La presente investigación tiene como propósito detectar el impacto del uso de internet sobre las actitudes políticas de las y los jóvenes como el interés en la política, eficacia política interna y confianza en las instituciones políticas y de gobierno en la Zona Metropolitana de Monterrey Nuevo León (ZMM), en el escenario preelectoral de 2018. Es importante destacar que "los jóvenes son agentes clave en el proceso de renovación de la sociedad, pues constituyen la generación que se hará cargo de la conducción del país" (Martínez, Silva \& Hernández, 2010, p. 26). En lo que corresponde a México, se puede indicar que algunos de los factores que incide en el compromiso político de los jóvenes son los medios de comunicación, internet y las redes sociales (Díaz \& Muñiz, 2017). En particular, el tipo de comunicación política que representa el uso de internet permite a sus usuarios aproximarse de manera directa a la información política, además de facilitarles la interacción de forma inmediata con otros individuos.

En el contexto donde se llevó a cabo el estudio, la ZMM, los jóvenes representan el 28.75\% (a marzo de 2019) de la lista nominal, lo que indica el número de ciudadanos que cuentan con credencial de elector vigente. Es decir, representa una parte muy importante de la población que puede participar en materia política. A su vez, el trabajo se realizó en el escenario preelectoral de 2018 en México, donde se celebraron elecciones a nivel federal para Presidente de la República, Senadores y Diputados Federales; en lo que corresponde al estado de Nuevo León se agregaron las elecciones para Diputados Locales y Ayuntamientos Municipales. Es decir, este constituyó un momento de movilización política en que se realizaban las campañas electorales para ocupar los diferentes puestos de elección popular antes mencionados.

El estudio tiene como objetivos de investigación identificar los niveles de uso de internet en materia política, de interés en la política, de eficacia política interna y de confianza en las instituciones políticas y de gobierno. Además, se tiene la intención de determinar sí el uso de internet en lo que corresponde al área política explica las diferentes actitudes políticas de los jóvenes en la ZMM.

Para la presente investigación, en primera instancia se presenta el marco teórico donde se tocan los temas de comunicación política, en lo particular del uso de internet con fines políticos. Asimismo, se desarrollan los conceptos de interés en la política, eficacia política interna y confianza en las instituciones políticas y de gobierno. En una segunda etapa se muestra la metodología que se utilizó para la aplicación del instrumento cuantitativo. Después se muestra el análisis de resultados en correspondencia a las variables, para finalmente presentar la discusión y conclusiones en relación con los objetivos del estudio e hipótesis de investigación. 


\section{Marco teórico}

En las actuales democracias representativas, donde son los ciudadanos quienes eligen a sus representantes y tratan de incidir en la toma de decisiones de éstos, es indispensable una ciudadanía que se encuentre bien informada sobre el acontecer político. Por ello, el presente trabajo hace énfasis en la manera en que los ciudadanos tienen acceso a dicha información. Es en este sentido que se ubica dentro del área de la comunicación política que puede ser definida "como el intercambio y la confrontación de los contenidos de interés público - político que produce el sistema político, el sistema de los medios y el ciudadano - elector" (Mazzoleni 2010, p. 36) o "como el conjunto de los mensajes que circulan en el interior de un sistema político y que condicionan su entera actividad, desde la formación de las demandas hasta los procesos de conversión del propio sistema” (Bobbio, Matteucci \& Pasquino, 1981, p. 264).

La comunicación política puede ser considerada como el enlace entre los diferentes actores políticos que permite fluir la información de contenido político entre ellos. Es decir, "facilita el proceso de información en cuanto a su alcance, significado y posibilidades, y es fundamental para el funcionamiento de los sistemas políticos" (Reyes Montes, 2007, p. 114). Se trata, por tanto, del "intercambio de mensajes entre los componentes del sistema político, es decir, entre todos los miembros de una sociedad" (Yanes Mesa, 2007, p. 359).

Existen diferentes teorías en relación con el impacto que podrían generar internet y las redes sociales con respecto a las actitudes políticas u orientaciones de los ciudadanos. Un primer posicionamiento indica que los procesos de comunicación política representados por la internet inciden de manera negativa en el comportamiento cívico del ciudadano. Dentro de esta postura encontramos lo planteado por Putnam (1995, 2000), que se ubica dentro las teorías del malestar mediático, quien señala que existe una desmovilización política entre los individuos que dedican su tiempo libre al consumo de programas de televisión y al uso de internet, e. El autor considera que al dedicarle un mayor tiempo a los medios de comunicación mencionados, las personas brindarán un menor tiempo para la formación de capital social, lo que se verá reflejado en la reducción de la capacidad para movilizarse políticamente.

Otra postura que se sitúa dentro de las teorías de la movilización política es la que propone Norris en su "Teoría del Círculo Virtuoso", donde expone que "las personas que ven más informativos de televisión, leen más periódicos, usan internet y prestan atención a campañas electorales, tienen consistentemente más conocimiento, confianza en el gobierno y son más participativos" (Norris, 2001, p. 25); es decir, son los más informados los que tendrán mayores niveles de conocimiento, confianza y participación política. A su vez, dentro de estas teorías se encuentra la postura de Delli Carpini (2000), quien indica que el uso de internet no solamente podría movilizar a los más interesados en política, sino que también tendrá un efecto positivo en los ciudadanos que no muestran compromiso 
político, incrementando su interés y motivándolos a participar. La presente investigación se concentrará en la postura propuesta desde el paradigma de la movilización política, considerando que el uso de internet incrementará el interés en la política, la sensación de eficacia política interna y la confianza en las instituciones de políticas y de gobierno.

En relación con lo anterior cabe destacar que el uso de internet se presenta como un nuevo paradigma que permite una comunicación horizontal, es decir, de persona a persona, sin intermediarios. Lo que a su vez se confronta con los medios de comunicación tradicionales (prensa escrita, radio y televisión) que simbolizan el modelo de la era analógica donde éstos son los intermediarios entre la información y el receptor de dicha información. Es importante comentar que existen diversas investigaciones que han tratado de marcar las diferencias entre ambos modelos, además de las ventajas del uso de internet en materia política y su relación con las actitudes políticas (Campos Freire, 2008; Córdula Almeida, Barboza de Sousa Piantavinha, \& Bispo Dos Santos, 2015; Delli Carpini, 200o; Kenski \& Jominie, 2006; Lobera \& Rubio, 2015; Meneses \& Bañuelos, 2009; Pérez, Peña, Genaut, Iturregui, \& Mendiguren, 2015; Yanez, 2007 ).

En el presente estudio nos enfocaremos exclusivamente al uso de internet en materia política dejando fuera de la investigación las implicaciones de los medios de comunicación tradicionales sobre las actitudes políticas de los jóvenes. Para abordar el tema de internet primeramente se podría comentar que no solamente permite la búsqueda de información en cualquier momento, sino que también ayuda a los usuarios a profundizar en los problemas mediante el uso de hipervínculos y motores de búsqueda. Es decir, internet permite a los ciudadanos incrementar los volúmenes de información y abundar sobre estos temas, dándole ventaja sobre los otros medios de comunicación (Kenski \& Jominie, 2006).

El uso de internet fomenta la interacción directa y el intercambio de mensajes entre sus usuarios, generando una nueva forma de acceso a la información que ayuda a disminuir las distancias geográficas y los tiempos para informarse. A su vez, internet puede reducir los costos de participación con relación al tiempo y esfuerzo y de esta manera se podría incrementar el acceso a mayor información (Boulianne, 2009). En este sentido, internet representa un nuevo modelo que incrementa la rapidez con la que la información puede ser guardada y transmitida, es decir, la facilidad de acceso permite generar oportunidades de interacción entre los ciudadanos de la red, eliminando las distancias representadas por el espacio geográfico, lo que motiva e incrementa las intenciones del ciudadano para involucrarse en la vida pública (Delli Carpini, 2000).

Delli Carpini (2000) comenta que es necesario hacer una distinción entre quiénes son los actores reales o potenciales en el uso de internet, e. Es en este sentido que menciona que las élites políticas (candidatos, grupos de interés o medios de comunicación) podrían utilizar internet para generar nuevas redes que les permitan acceder a nuevas audiencias. Por otro lado, encontramos a los 
ciudadanos más comprometidos, a los que internet les ayuda a la reducción de costos en sus compromisos, favoreciendo la realización de diferentes tipos de actividades en la materia. Finalmente, Delli Carpini (2000) comenta que este medio puede motivar a participar a los ciudadanos que no sienten compromiso; en lo particular indica que en los adultos jóvenes la red sería una forma de incrementar el interés transformándolo en acción política.

La nueva realidad que representan las Tecnologías de la Información y de la Comunicación (TIC) están creando un nuevo espacio mediático que de manera importante se refleja en cambios en el contexto social (Campos Freire, 2008; Pérez et al., 2015). En relación con lo anterior, se puede indicar que el tipo de comunicación horizontal que se genera con el uso de internet y las redes sociales permite a los ciudadanos fundar sus propias representaciones en relación con el sistema político y sus actores, ya que el intercambio de información y opiniones se puede dar de manera directa sin necesidad de intermediarios; es decir, los ciudadanos construyen sus propias perspectivas, por medio del intercambio de opiniones e influencias, que incidirán de manera importante en sus actitudes políticas afuera de la red (Lobera \& Rubio, 2015). Asimismo, "internet es un medio interactivo que multiplica las posibilidades de contacto de una forma muy eficiente en términos de inversión en tiempo y crea un entorno intensivo de comunicación” (Borge, Cardenal \& Malpica, p. 733).

Esta nueva realidad dentro del espacio de la comunicación política creada por internet, como medio interactivo de comunicación, ha generado cambios importantes en la sociedad, ya que les permite a los ciudadanos la opción de tener acceso a una gran cantidad de fuentes alternas de información, incrementando su libertad de expresión, además de reducir las distancias frente a los dirigentes políticos. Es decir, las redes sociales podrían permitir un espacio para que los desencantados con la política puedan expresar su pensamiento (Moya Sánchez \& Herrera Damas, 2015b).

Internet permite una representación de enfoques multidireccionales generando un esquema donde "muchos informan a muchos", a diferencia del modelo tradicional (prensa escrita, radio y televisión) donde "pocos informan a muchos" (Córdula Almeida et al., p.88). Es en este sentido que diferentes estudios indican que el uso de internet y las redes sociales genera los espacios que permiten empatar las posibilidades de interacción de los diferentes actores políticos, ya que pueden participar en igualdad de condiciones (Amado \& Tarullo, 2015; Moya Sánchez \& Herrera Damas, 2015a).

Dentro de las actitudes políticas en las cuales el uso de internet tendrá un efecto positivo se encuentre el interés en la política, que puede definirse como el equivalente al nivel en que el ciudadano estaría dispuesto a acceder y procesar la información que se relaciona con temas políticos (Galais, 2012). Es decir, "el interés que posee un sujeto o grupo sobre los asuntos políticos, o al menos, sobre los resultados de estos" (Brussino, Rabbia \& Sorribas, 2009, p. 281). Se podría decir que el interés en la política es la intención que tiene una persona de procesar toda aquella información que se relacione con el ámbito político. En relación con lo anterior, “el interés por la política tiene una 
relevancia fundamental en la implicación global de la ciudadanía en la política, llegando a considerarse, a nivel normativo, un prerrequisito importante de la política democrática” (Verge \& Tormos, 2012, p.90).

Otra actitud hacia la política sería la sensación de eficacia política que podría ser considerada por un individuo como la sensación positiva de oportunidad ofrecida por el sistema político para incidir en cuestiones políticas. De acuerdo con Brussino et al. (2009), ésta se puede dividir en eficacia política interna, considerada como "la capacidad auto-percibida de participación y competencia en asuntos políticos", y eficacia política externa, definida como "la creencia que posee un sujeto, referida a la capacidad que manifiesta el gobierno para dar respuesta a la intención de este ciudadano para influir en aspectos gubernamentales" (p. 282).Por otra parte, Mazzoleni (2010) considera que "uno de los determinantes psicológicos de la participación es la sensación de eficacia que tenga o no el ciudadano, que depende a su vez del grado de apertura que percibe en el sistema político, de la capacidad de respuesta del sistema a sus impulsos participativos" (p. 286).

Dentro de las actitudes estudiadas se encuentra la confianza en las instituciones políticas y de gobierno, que puede ser considerada como el juicio evaluativo que presenta un ciudadano en relación con lo que él considera que debería ser el actuar de una determinada institución. Así, "una persona puede confiar en que una institución hará lo que se cree que debería hacer, o que va a comportarse de la manera que se espera de ella" (Segovia, Hayde, González, Manzi, \& Carvacho, 2008, p. 42). Por tanto, "la confianza política es entendida como un juicio sobre el funcionamiento de las instituciones políticas, en particular, y sobre el sistema político, en general” (Rivera, 2019, p. 562). Los niveles de confianza que el ciudadano presenta frente a las instituciones políticas exponen sus experiencias con éstas, además de sus orientaciones sociales y culturales (González de la Vega, Quintanilla \& Tajonar, 2010).

La confianza política es una evaluación hacia las instituciones que, de ser positiva, le daría legitimidad democrática a un gobierno. Asimismo, es relevante comentar que no evaluar o una confianza total en las instituciones de gobierno podría poner en peligro la estabilidad democrática ya que, en este sentido, los ciudadanos renuncian a la esperanza de que el sistema político funcione de manera correcta o a su rol de ciudadano vigilante de la actuación de dichas instituciones. Se podría indicar que cierto nivel de confianza y escepticismo a la vez sería una mezcla deseable para una democracia (Gershtenson, Ladewig \& Plane, 2013).

En consideración al tema y los objetivos de la presente investigación, y con base en la información expuesta en el marco teórico, se proponen las siguientes preguntas e hipótesis de investigación:

PI1: ¿Cuál es el nivel de uso de internet en materia política por parte de las y los jóvenes? PI2: ¿Cuál es el grado interés en la política de las y los jóvenes? 
PI3: ¿Cuál es la sensación de eficacia política interna por parte de las y los jóvenes?

PI4: ¿Cuál es el nivel de confianza de las y los jóvenes en las instituciones políticas y de gobierno?

PI5: ¿De qué manera influye el uso de la internet con fines políticos en la confianza en las instituciones políticas y de gobierno de las y los jóvenes?

HI1: La conversación política interactiva tiene un impacto positivo en el interés hacia la política.

HI2: El consumo de contenido político online produce un mayor interés en la política.

HI3: La conversación política interactiva tiene un efecto positivo en la sensación de eficacia política.

HI4: El consumo de contenido político online influye en una mayor sensación de eficacia política.

\section{Metodología}

Para el estudio de se aplicó de manera aleatoria una encuesta de opinión representativa de las y los jóvenes (18 a 29 años) en los diferentes municipios que conforman la Zona Metropolitana de Monterrey, en el estado mexicano de Nuevo León (ZMM) durante el mes de mayo del año 2018 previo a las elecciones federales en México que se celebraron el primero de julio de este mismo año. El tamaño de la muestra fue de 1,018 jóvenes, aplicando un nivel de confianza del 95\% y un margen de error muestral de 2.35\%. Para medir el nivel de confianza y el margen de error, se manejó la fórmula de población infinita, quedando de la siguiente manera:

$$
n=\left[\left(Z_{\alpha} / 2\right]^{2}(p)(q)\right] / 2 \text { despejando } n \text {, resulta: } E=\left[\left(Z_{\alpha} / 2\right]^{2}(p)(q)\right] / n
$$

En relación con el perfil de la muestra, se puede comentar que el 52.3\% fueron mujeres y el 47.7\% son hombres. Los ingresos que los encuestados manifestaron indican que el 34.7\% reportó que tenían un ingreso no mayor a los \$10,000, el 11.1\% comentó que su ingreso era mayor a los \$10,000 pero no sobrepasa los $\$ 30,000$ y el $2 \%$ comentó tener ingresos mayores a los $\$ 30,000$. Finalmente, el 37.8\% indicó que no trabajaban. Por otra parte, en lo que corresponde al nivel educativo, los encuestados revelaron que el 6.1\% tenía terminado un nivel básico (hasta educación secundaria), el 59\% indicó tener terminado el nivel medio superior (preparatoria o bachillerato), el 30.9\% reportó haber 
finalizado la universidad (profesionistas) y el 1.7\% comentó tener posgrado terminado. El 80.7\% de los jóvenes indicó tener una edad entre 18 y 24 años, y el 19.3\% entre 25 y 29 años.

\section{Cuestionario}

Para la elaboración del cuestionario se tomó como propuesta varias escalas de investigaciones realizadas previamente, adaptándolas a las necesidades del estudio.

\section{Variables de control}

Estas variables fueron de corte sociodemográfico, por lo que se les preguntó a los encuestados su edad, el nivel de ingreso mensual personal, donde (1) era menos de 6,00o pesos mexicanos y (4) más de 30,001 pesos mexicanos, y el nivel educativo, donde (1) era que no tiene y (7) era posgrado.

\section{Variables independientes}

Para la creación del constructo conversación política interactiva se tomó como referencia la escala propuesta por Shah et al. (2007), con una escala Likert donde (1) era nada y (5) era bastante. Se preguntó a las y los jóvenes en relación con las elecciones 2018 qué tanto realizaron actividades como hacer y subir a internet vídeos, animaciones, audios, fotos u otro producto audiovisual para expresar sus posturas políticas, compartir noticias, videos o posts sobre política por internet, o participar en discusiones políticas a través de la red. El análisis factorial realizado dio como resultado un KMO de .739, la medida de adecuación muestral (MSA) marcó por arriba de o.6, y las comunalidades estuvieron por encima de 0.5. Por otra parte, la varianza acumulada fue del 80.68\%. Con relación al análisis de fiabilidad, se obtuvo un Alfa de Cronbach ( $\alpha$ ) de .879 (Ver Tabla 1).

En la construcción de la variable de consumo de contenido político online se les preguntó a los participantes, considerando una escala de Likert donde (1) era nada y (5) era bastante, qué tanto buscaban información como propuestas de campaña, actores políticos, líderes de opinión (analistas), casos de corrupción, candidatos y partidos políticos en internet. Esta escala se tomó considerando el estudio presentado por la Asociación de Internet.mx sobre los "Hábitos de los Usuarios de Internet en México 2017”. En lo que respecta el análisis factorial, se reportó un KMO de .826, la medida de adecuación muestral (MSA) se presentó por encima de o.6, las comunalidades fueron por arriba de o.5 y la varianza acumulada fue del 67.90\%. En relación con el análisis de fiabilidad, se observó un Alfa de Cronbach ( $\alpha$ ) de .896 (Ver Tabla 1). 


\section{Variables dependientes}

Para la medición de la variable interés en la política se utilizó la escala presentada por Muñiz y Maldonado (2011), donde se les cuestiona a los encuestados, a través de una escala de Likert de 5 puntos donde (1) representa nada y (5) representa bastante, sobre su nivel de interés en la política municipal, estatal, nacional e internacional. En el análisis factorial se presentó un KMO de .701, la medida de adecuación muestral (MSA) fue mayor a o.6, las comunalidades quedaron por encima de 0.5 y la varianza acumulada fue del 78.38\%. A su vez, el análisis de fiabilidad reveló un Alfa de Cronbach ( $\alpha$ ) de .906, ligeramente por encima de los parámetros permitidos por lo que se dejó la variable tal cual se construyó (Ver Tabla 1).

Tabla 1. Análisis factorial y de fiabilidad con relación a las variables independientes y dependientes

\begin{tabular}{cccc}
\hline Variables o constructos & KMO & Alfa de Cronbach $\alpha$ & Varianza acumulada \\
\hline Conversación política interactiva & .739 & .879 & $80.68 \%$ \\
\hline Consumo de contenido político online & .826 & .896 & $67.90 \%$ \\
\hline Interés en la política & .701 & .906 & $78.38 \%$ \\
\hline Eficacia política interna & .735 & .854 & $77.98 \%$ \\
\hline $\begin{array}{c}\text { Confianza en las instituciones políticas } \\
\text { y de gobierno }\end{array}$ & .801 & .892 & $77.36 \%$ \\
\hline
\end{tabular}

Fuente elaboración propia

Para la medición de la eficacia política interna se utilizó una escala de Likert, donde (1) era nada y (5) bastante, tomando como referencia la propuesta de ENCUP (2012). Se consideró no solamente la influencia en las decisiones de gobierno, sino también en un proceso electoral, preguntando a las y los jóvenes qué tanto consideraban que su voto podría hacer la diferencia en una elección, si realmente tenían algo que decir en relación con lo que los gobernantes deciden y, finalmente, si ellos pensaban que podrían marcar la pauta participaban en los procesos electorales. Al llevarse a cabo el análisis factorial, éste dio como resultado un KMO de .735, la medida de adecuación muestral (MSA) estuvo por encima 0.6 , las comunalidades fueron mayores de $0.5 \mathrm{y}$ la varianza acumulada fue del 77.98\%. El análisis de fiabilidad reportó un Alfa de Cronbach ( $\alpha$ ) de .854 (Ver Tabla 1).

En lo que corresponde a la confianza en las instituciones políticas y de gobierno, se utilizó la propuesta de la ENCUP (2012), con una escala de Likert donde (1) era nada y (5) era bastante, preguntando a los encuestados su nivel de confianza en las siguientes instituciones: partidos políticos, presidente de la República, Suprema Corte de Justicia de la Nación (SCJN), Diputados y Senadores. $\mathrm{El}$ análisis factorial reveló un KMO de .801, la medida de adecuación muestral (MSA) estuvo por 
arriba de 0.6, las comunalidades fueron mayores de $0.5 \mathrm{y}$ la varianza acumulada fue del 77.36\%. La fiabilidad mostró un Alfa de Cronbach ( $\alpha$ ) de .892 (Ver Tabla 1).

\section{Análisis de resultados}

La variable conversación política interactiva $(M=1.94, D E=0.887)$ en general presentó una presencia baja entre la muestra, por debajo de la media promedio. La actividad que con mayor frecuencia realizan las y los jóvenes fue compartir noticias, videos o posts sobre política por internet $(M=2.37$, $D E=1.26$ ), aunque cabe mencionar que sólo el $19 \%$ indicó que lo realizaba con frecuencia (mucho bastante). Por otro lado, las actividades como hacer y subir a internet vídeos, animaciones, audios, fotos $\mathrm{u}$ otro producto audiovisual para expresar sus posturas políticas ( $M=1.66, D E=1.03)$ y participar en discusiones políticas a través de la red $(M=1.94, D E=0.887)$ fueron las actividades que se llevaron a cabo con menor periodicidad.

El consumo de contenido político online como constructo fue bajo $(M=2.80, D E=0.946)$, ya que el valor se situó por debajo de la media aritmética. Sin embargo, la actividad que realizaron los encuestados con mayor asiduidad en internet fue el seguimiento a información sobre candidatos ( $M$ = 3.33, $D E=1.22$ ), reportándose por encima de la media promedio. En este sentido, el $48.6 \%$ (mucho -bastante) de las y los jóvenes comentó que lo realiza con mucha frecuencia. Por otra parte, a la atención a este tipo de información le siguió la búsqueda de información sobre partidos políticos $(M=$ $2.96, D E=1.27)$ y de casos de corrupción $(M=2.90, D E=1.22)$. En la primera actividad el $35.2 \%$ (mucho - bastante) mencionó que lo llevaba a cabo con frecuencia y en la segunda el 33\% (mucho bastante). Finalmente, la atención a líderes de opinión $(M=2.36, D E=1.17)$ y actores políticos en general $(M=2.57, D E=1.18)$ fue la información a la que menos atención prestaron.

Por otra parte, el comportamiento del interés en la política como variable fue positivo $(M=$ $3.31, D E=0.945$ ), ya que se reporta por encima de la media promedio. A su vez, el mayor interés de las y los jóvenes se presentó de una manera importante en la política nacional ( $M=3.76, D E=1.13$ ), donde el 65.9\% (mucho - bastante) comentó tener un interés relevante. Asimismo, este interés es seguido por el mostrado en la política internacional $(M=3.29, D E=1.33)$ y la nacional $(M=3.19, D E$ =1.12). Es importante comentar que el menor interés se presentó con respecto a la política municipal $(M=3.02, D E=1.13)$.

El sentimiento de eficacia política interna como constructo tuvo una presencia importante ( $M$ $=3.28, D E=1.09$ ), ya que se presenta por arriba de la media aritmética. El mayor nivel de eficacia que reportaron los encuestados fue considerar que su voto podría hacer la diferencia en una elección ( $M=3.51, D E=1.18$ ), pues el 52.2\% (mucho - bastante) se manifestó en este sentido. A su vez, este tipo de sentido de eficacia política fue seguida por creer que su participación en procesos electorales podría marcar la diferencia $(M=3.28, D E=1.54)$. Finalmente, los y las jóvenes indicaron que tenían 
algo que decir en lo que los gobernantes deciden $(M=3.07, D E=1.54)$, es decir, podían influir en la toma de decisiones de la autoridad.

La confianza en las instituciones políticas y de gobierno como variable tuvo una presencia bastante baja $(M=2.17, D E=0.783)$, menor a la media aritmética. En relación con la mayor confianza se reportó frente a la SCJN $(M=2.53, D E=1.09)$, aunque es importante destacar que sólo el 17.2\% (mucho - bastante) manifestó que era importante. Los índices menores de confianza se tuvieron frente a instituciones como los partidos políticos $(M=1.94, D E=0.896)$, presidente de la República $(M=2.10, D E=1.35)$ y diputados y senadores $(M=2.12, D E=0.953)$.

Para dar mayor relevancia a los resultados descriptivos en relación con los niveles de conversación política interactiva, interés en la política eficacia política interna y confianza en instituciones políticas y de gobierno, se aplicó la prueba $t$ de Student de muestras independientes. Para ello se tomó como base el estudio previo "La participación política convencional de los jóvenes en el Estado de Nuevo León. Factores que explican esta participación en el escenario poselectoral 2015" financiado por el Programa para el Desarrollo Profesional Docente, para el tipo superior (PRODEP) realizado en el mismo contexto y población (ZMM en jóvenes de 18 a 29 años) en el año 2015. Para ello, las variables se construyeron tomando en consideración los mismos ítems para ambos estudios (que se presentaron en el apartado de metodología).

Tabla 2. Resultados descriptivos

\begin{tabular}{cccccc}
\hline Variables o constructos & \multicolumn{2}{c}{ Media } & \multicolumn{2}{c}{ Desviación estándar } & $t$ de Student \\
\hline & 2015 & 2018 & 2015 & 2018 & \\
\hline Conversación política interactiva & 1.90 & 1.94 & .876 & .887 & n.s. \\
\hline $\begin{array}{c}\text { Consumo de contenido político on- } \\
\text { line }\end{array}$ & - & 2.80 & - & .946 & n.a. \\
\hline $\begin{array}{c}\text { Interés en la política } \\
\text { Eficacia política }\end{array}$ & 3.02 & 3.31 & .933 & .945 & $-5.358 p<.001$ \\
\hline $\begin{array}{c}\text { Confianza en las instituciones polí- } \\
\text { ticas y de gobierno }\end{array}$ & 3.03 & 3.28 & .962 & 1.09 & $-5.603 p<.001$ \\
\hline $\begin{array}{c}\text { Nota: } \\
\text { lo que correspon }\end{array}$ & 1.97 & 2.17 & .747 & .783 & $-5.839 p<.001$ \\
\hline
\end{tabular}

Nota: En lo que corresponde a la variable consumo de contenido político online, no se trabajó con la misma en la encuesta 2015, por lo que no es posible aplicar con ella una prueba de contraste. Fuente Elaboración propia

Después de realizar la prueba $t$ de Student, se pudo determinar que el incremento en los niveles de interés en la política, de eficacia política y de confianza en las instituciones políticas y de gobierno fue estadísticamente significativo $(p<.001)$, pero no así el aumento en la conversación política interactiva. En relación con el consumo de contenido político online, no se midió ya que esta variable no fue utilizada en la encuesta aplicada en el año 2015. 
$\mathrm{Al}$ dar por concluidos los análisis descriptivos, se buscó la relación o covarianza entre las variables socioeconómicas con las variables dependientes interés en la política, eficacia política interna y confianza en las instituciones políticas y de gobierno. Para esto se utilizó la prueba $r$ de Pearson. La relación entre la variable de control edad y el interés en la política presentó una relación negativa ( $r$ $=-.076, p=.016$, con la sensación de eficacia política interna no se reportó correlación y con la variable confianza en instituciones políticas y de gobierno fue negativa $(r=-.087, p=.005$. Por otra parte, en lo que corresponde al nivel de ingresos y educativo no se mostró ninguna relación con las variables dependientes.

Al finalizar el análisis de correlación de las variables de control se pasó al estudio de la relación entre las variables independientes conversación política interactiva y consumo de contenido político online con las variables dependientes interés en la política, eficacia política interna y confianza en las instituciones políticas y de gobierno. Para esto se utilizó la prueba $r$ de Pearson. En la tabla 3 se muestra la relación entre la conversación política interactiva con las variables dependientes interés en la política, eficacia política y confianza en las instituciones políticas y de gobierno. Se puede indicar que la conversación política interactiva tuvo una relación positiva con la variable independiente interés en la política $(r=.344, p<.001)$. Asimismo, la relación con la eficacia política interna $(r=.210, p$ $<$.oo1) fue también positiva. Finalmente, la correlación con las instituciones políticas y de gobierno $(r=.121, p<.001)$ fue igualmente importante.

Tabla 3. Correlaciones entre las variables de control e independientes con las dependientes

\begin{tabular}{ccccccc}
\hline Variables & 1 & 2 & 3 & 4 & 5 & 6 \\
\hline Interés en la política & - & $.253^{* *}$ & $.241^{* *}$ & $-.076^{*}$ & $.344^{* *}$ & $.490^{* *}$ \\
\hline $\begin{array}{c}\text { Eficacia política interna } \\
\text { Confianza en instituciones políticas y de } \\
\text { gobierno }\end{array}$ & - & $.198^{* *}$ & .046 & $.210^{* *}$ & $.270^{* *}$ \\
\hline Edad & & - & $-.087^{* *}$ & $.121^{* *}$ & $.203^{* *}$ \\
\hline Conversación política interactiva & & & - & $-.071^{*}$ & $.092^{* *}$ \\
\hline
\end{tabular}

Consumo de contenido político online

$-$

Nota: $\mathrm{N}=1018$ casos. Los resultados fueron estadísticamente significativos a un nivel de confianza de ${ }^{*} p<.05 ;{ }^{* *} p<.01 ;{ }^{* * *} p$ $<$.001. Fuente elaboración propia.

En la tabla 3 también se puede observar la relación entre el interés en la política, la eficacia política interna, la confianza en las instituciones políticas y de gobierno y el consumo de contenido político online. Se puede indicar que la atención a información política en internet se relacionó de manera positiva con el interés en la política $(r=.490, p<.001)$. A su vez, la relación con la eficacia 
política $(r=.270, p<.001)$ es positiva, al igual que con la confianza en las instituciones políticas y de gobierno $(r=.203, p<.001)$.

En la última etapa de análisis, y tomando en consideración las correlaciones anteriores, se buscó determinar el nivel explicativo de la edad, la conversación política interactiva y el consumo de contenido político online sobre las variables dependientes interés en la política, eficacia política interna y confianza en las instituciones políticas y de gobierno que revelaron asociación de acuerdo con la prueba $r$ de Pearson.

Para el primer modelo se tomó como variable dependiente al interés en la política y como variable de control la edad, seguida por las variables independientes la conversación política interactiva y el consumo de contenido político online. Se reportó que la variable de control edad no tenía ningún nivel explicativo sobre la dependiente. Por otra parte, las variables independientes explicaron el $26.1 \%$ de la varianza $\left(R^{2}=.261\right)$, éstas cumplen con el supuesto de independencia respecto a la dependiente, ya que el valor del test Durbin - Watson es de $d=1.85$ encontrándose dentro de los parámetros reconocidos. El nivel explicativo de la conversación política interactiva (con un valor de $\beta=.124 ; p<.001$ ), fue positivo, por lo que, a niveles más altos de comunicación política interactiva se generó un mayor interés en la política. A su vez, el nivel explicativo del consumo de contenido político online (con un valor de $\beta=.437 ; p<.001$ ) fue relevante en sentido positivo, es decir, un mayor consumo de contenido político online se incrementaron los niveles de interés en la política.

Tabla 4. Nivel explicativo de las variables socioeconómicas e independientes comunicación política interactiva y la atención a noticias en internet sobre las variables dependientes

\begin{tabular}{|c|c|c|c|c|}
\hline Variable & Edad & $\begin{array}{l}\text { Conversación po- } \\
\text { lítica interactiva }\end{array}$ & $\begin{array}{c}\text { Consumo de contenido } \\
\text { político online }\end{array}$ & $R^{2}$ \\
\hline $\begin{array}{c}\text { Modelo 1 } \\
\beta \\
\text { Interés en la política }\end{array}$ & - & $.124^{* * *}$ & $.437^{* * *}$ & .261 \\
\hline $\begin{array}{c}\text { Modelo } 2 \\
\beta \\
\text { Eficacia política } \\
\end{array}$ & - & $.099^{* * *}$ & $.224^{* * *}$ & .082 \\
\hline $\begin{array}{c}\text { Modelo } 3 \\
\beta \\
\text { Confianza en las institu- } \\
\text { ciones políticas y de go- } \\
\text { bierno } \\
\end{array}$ & - & - & $.194^{* * *}$ & .043 \\
\hline
\end{tabular}

En el segundo modelo se tomó como variable dependiente a la eficacia política interna, como variable de control la edad y como variables independientes la conversación política interactiva y el consumo de contenido político online. Se observó que la variable de control edad no marcaba ningún 
nivel explicativo sobre la dependiente, con relación a las independientes explican el $8.2 \%$ de la varianza $\left(R^{2}=.082\right)$, éstas cumplen con el supuesto de autonomía respecto a la dependiente, ya que el valor del test Durbin - Watson es de $d=1.70$ estaba dentro de los parámetros permitidos. El nivel explicativo de la conversación política interactiva (con un valor de $\beta=.099 ; p<.001$ ) fue importante. En este sentido, un grado alto de conversación política interactiva, provocó una mayor sensación de eficacia política interna. En el mismo sentido, el nivel explicativo del consumo de contenido político online (con un valor de $\beta=.224 ; p<.001$ ) fue positivo, por lo que, un mayor consumo de contenido político online produjo niveles más altos de sensación de eficacia política.

En relación con el tercer modelo, se tomó como variable dependiente a la confianza en instituciones políticas y de gobierno y como variable de control la edad y como variables independientes la conversación política interactiva y el consumo de contenido político online. En relación con la variable de control, ésta no mostró ningún nivel explicativo respecto a la variable dependiente. En lo que corresponde a las independientes, solo el consumo de contenido político online explicó el 4.3\% de la varianza $\left(R^{2}=.043\right)$, ya que la variable conversación política interactiva no presentó ningún nivel explicativo. La variable independiente consumo de contenido político online cumplió con el supuesto de independencia respecto a la dependiente, ya que el valor del test Durbin - Watson fue de $d$ $=1.76$ reportándose dentro de los parámetros reconocidos. El nivel explicativo del consumo de contenido político online (con un valor de $\beta=.194 ; p<.001$ ) fue positivo, por lo que, un mayor consumo de contenido político online incrementó los niveles de confianza en instituciones políticas y de gobierno.

\section{Discusión y conclusiones}

Los resultados de la investigación en primer lugar permiten dar cumplimiento a los objetivos que se plantearon, ya que se pudo identificar los niveles de uso de internet en materia política por parte de las y los jóvenes. Esto se determinó mediante las variables conversación política interactiva y consumo de contenido político online, donde se puede indicar que son bajos. En este sentido, se puede mencionar que en materia política el uso de internet es limitado en comparación con los niveles de consumo que reporta la Encuesta Nacional sobre Disponibilidad y Uso de Tecnologías de la Información en los Hogares (ENDUTIH, 2017) realizada por el INEGI, donde se determina que el 83.7\% de las y los jóvenes de 18 a 34 años utilizan este medio para la realización de actividades diversas donde predominan el entretenimiento, la comunicación, el acceso a contenidos audiovisuales y acceso a redes sociales.

Por otra parte, se puede mencionar que se identificó que los niveles de interés en la política y sentimiento de eficacia política interna son, de alguna manera, importantes desde una perspectiva 
positiva, más no necesariamente demasiado altos. Es decir, en las y los jóvenes existe un interés y un sentimiento de eficacia política interna positiva. Con relación a la confianza en las instituciones políticas y de gobierno, se pudo identificar que ésta es baja, por lo que se podría decir que las y los jóvenes no consideran que las instituciones evaluadas realmente estén cumpliendo con su deber ser.

Finalmente, como objetivo importante se planteó el determinar el nivel explicativo de uso del internet en materia política en relación con las actitudes políticas planteadas en el estudio de las y los jóvenes. En este sentido, sí se pudo establecer que las variables que sirvieron para la medición de uso de internet con fines político, como son la conversación política interactiva y consumo de contenido político online, sí presentan un nivel explicativo positivo con relación al interés en la política y el sentimiento de eficacia política interna. En relación con la confianza en las instituciones políticas y de gobierno, sólo la variable consumo de contenido político online demostró tener un nivel explicativo significativo.

Para dar respuesta a las preguntas de investigación relacionadas con los resultados descriptivos se tomó en consideración el estudio previo ya mencionado realizado en el escenario postelectoral 2015, en el mismo contexto (ZMM) y con jóvenes de la misma edad (18 a 29 años), utilizando la prueba de $t$ de Student para muestras independientes, permitiéndonos determinar sí existe cambio en los niveles de interés en la política, eficacia política interna y de confianza en las instituciones políticas y de gobierno, así como si estos cambios son significativos.

Respecto a la primera pregunta de investigación, que planteaba ¿cuál es el nivel de uso de internet en materia política por parte de las y los jóvenes?, como ya se mencionó ésta se midió mediante dos variables. La primera la conversación política interactiva que a manera de analogía se puede comentar que prácticamente representaría a la conversación política interpersonal, pero desde un medio digital, ya que permite la interacción inmediata entre los usuarios. Es decir, internet permite una relación directa entre emisores-receptores, un intercambio de mensajes entre los actores políticos sin intermediarios (García Luengo \& Maurer, 2009). Y la segunda variable sería el consumo de contenido político online.

En lo que respecta a la primera variable, se pudo observar en relación con el estudio 2015 que no se presentó un incremento importante en los niveles de conversación política interactiva. Por otra parte, y con relación al consumo de contenidos políticos online, no se pudo comparar ya que en el estudio previo no se construyó dicha variable. Pero sí se puede comentar que al igual que la conversación política interactiva es bajo el consumo de contenidos políticos online por parte de las y los jóvenes. Lo anterior podría estar relacionado con la confianza que las y los jóvenes presentan frente a la internet, redes sociales y páginas Web, que de acuerdo con la Encuesta Nacional de Cultura Política de los Jóvenes (Gómez-Tagle, Tejera Gaona, Aguilar López, Ramírez, \& Díaz Jiménez, 2012) es baja. Unos resultados semejantes se presentan en el estudio que se realizó previamente en 2015 y en el 2018 en la ZMM, donde se indica que la confianza en las redes sociales es baja ya que se encuentra por debajo de la media promedio. 
En relación con la segunda pregunta de investigación, que planteaba ¿̇cuál es el grado interés en la política de las y los jóvenes?, se puede revelar que se encuentra en un punto positivo como ya se indicó, al confrontar con otros estudios como el presentado por Durand Ponte (1995) y la ENCUP (2012) a población abierta y el de la Encuesta Nacional de Valores en Juventud (2012) aplicada a jóvenes desde los 15 años. Los resultados de la investigación muestran niveles más altos de interés, aunque cabe mencionar que las muestras con las que se compara son diferentes con relación a la edad y al contexto en que se aplicó. Por otra parte, el estudio previo realizado 2015 permite hacer un comparativo en relación con edad y contexto, y al respecto se puede indicar que los niveles de interés en la política han incrementado de 2015 a 2018 de manera significativa. Posiblemente esto se podría relacionar con el interés en la campaña electoral a la Presidencia de la República pues, como lo indica Moreno (2009), el interés en las campañas "suele incrementarse conforme se desarrollan los periodos en los que los candidatos presidenciales y sus partidos tratan de movilizar el apoyo de los electores" (p. 212). A su vez, es importante señalar que el interés del ciudadano frente al sistema político es de especial trascendencia, ya que "le permitirá exponerse a lo que ocurre en el mismo sistema y obtendrá la información precisa para el desarrollo de su participación” (Saldierna, Marañon \& Muñiz, 2015, p. $150)$.

Al contestar la tercera pregunta del estudio, relativa a ¿̇cuál es la sensación de eficacia política interna por parte de las y los jóvenes?, se puede decir que esta se encuentra en un nivel relativamente positivo. $\mathrm{Al}$ hacer un comparativo con el estudio 2015, se puede observar un incremento relevante. Esto es importante ya que niveles más altos de sentimiento de eficacia política interna demuestran una mayor apertura por parte del sistema político. En este sentido, esta sensación puede ser considerada como un determinante psicológico para la participación del ciudadano (Mazzoleni, 2010).

Con relación a la cuarta pregunta, ¿cuál es el nivel de confianza de las y los jóvenes en las instituciones políticas y de gobierno?, como ya se comentó, ésta es bastante baja, aunque es importante destacar que ha incrementado de manera significativa con relación al estudio 2015 sin dejar de ser muy baja. En relación con estos resultados se puede argumentar que "la persistencia de las desigualdades, incluso en ambiente políticamente más abierto y competitivo, seguirá siendo un motivo importante, si no es que fundamental, de la desconfianza en las instituciones" (Moreno, 2010, p.25) en México. Aunque nuestro sistema electoral es más competitivo y confiable, las desigualdades sociales siguen siendo un problema que resolver.

En relación con la quinta pregunta, ¿de qué manera influye el uso de la internet con fines políticos en la confianza en las instituciones políticas y de gobierno de las y los jóvenes?, que es de corte explicativa, se puede reportar que los resultados indican que sólo el consumo de contenido político online muestra un nivel explicativo, más no contundente, en relación con la variable. A su vez, en la investigación la conversación política interactiva no afecta el nivel de confianza en las instituciones estudiadas. Esto podría contrastar con lo que indica Ceron (2015) en su estudio, donde considera que "la naturaleza no mediada y ascendente de las redes sociales de la Web 2.0 albergarán y 
favorecerán la circulación de información alternativa que afecta negativamente la confianza política” (p. 495). Pero podría aproximarse a la postura de Norris (2001), quien indica que el uso internet y la atención a las campañas electorales generarán mayor confianza en el gobierno.

En lo que respecta a la primera hipótesis de investigación, donde se asevera que la conversación política interactiva tiene un impacto positivo en el interés en la política, ésta es válida de acuerdo con los resultados obtenidos, al igual que la segunda que indica que el consumo de contenido político online produce un mayor interés en la política. Estas dos variables fueron utilizadas para medir el uso de internet en materia política. Es importante comentar que la facilidad que presenta internet para incrementar la velocidad con que la información puede ser trasmitida y la disposición del acceso a esta por parte del ciudadano, además de la oportunidad de interactuar de manera directa eliminando las distancias que representan los espacios geográficos, podría incrementar y motivar al ciudadano para involucrarse en la vida pública (Delli Carpini, 2000). Por ello, el uso de internet con fines políticos incrementaría el interés de las y los jóvenes en el sistema político, que podría generar una mayor participación de éstos en la política.

En lo que respecta a las afirmaciones presentadas en las hipótesis tres y cuatro, se puede reportar que son válidas ya que tanto la conversación política interactiva, pero sobre todo el consumo de contenidos online, tiene un nivel explicativo importante sobre la sensación de eficacia política interna por parte de las y los jóvenes. Es en este sentido que los resultados concurren con los obtenidos por Corduneanu y Muñiz (2011), quienes indican que "internet es el medio que más fuerte genera un sentimiento de eficacia política" (p. 305). Se podría decir que el uso de internet con fines políticos podría fomentar la percepción de las y los jóvenes con relación a su capacidad de influir en los asuntos políticos.

En conclusión, se podría indicar que las ventajas que presenta el internet y las redes sociales con relación a su capacidad de reducción de distancias y tiempos, además de facilitar el acceso directo a la información sin intermediarios, que a su vez le permite en este caso a las y los jóvenes a interactuar de manera directa podrían incrementar sus niveles de interés y eficacia política interna motivándolos a ser más activos en la política. Es decir, no solamente para elegir a sus representantes, sino también para incidir en la toma de decisiones de éstos. Pues, como indican Díaz y Muñiz (2017), en México los medios de comunicación, el internet y las redes sociales poseen un impacto notable en el compromiso político de las y los jóvenes.

Con relación a la desconfianza en las instituciones de gobierno, no es un problema que se presenta de manera aislada en México. Por ejemplo, en Estados Unidos de América el estudio "Public Trust in Government: 1958-2019" realizado por el Pew Research Center U.S. Politics and Policy (2019) indica que desde el año 2007 la confianza en el gobierno no supera el 30\%. A su vez, es importante destacar que en la década de los 90 cuando la economía creció se presentó un incremento en la confianza en el gobierno. Por otra parte, de acuerdo con el estudio presentado por Latinobarómetro 
"La confianza en América Latina 1995 - 2015. 20 años de opinión pública Latinoamérica”, la confianza en los partidos políticos, el congreso y el poder judicial del año 1995 al 2015 no supera el nivel de 4 de cada 10 ciudadanos.

En lo que respecta a la confianza en las instituciones políticas y de gobierno en México, se parte de la propuesta de Moreno (2009), quien indica que un estado con desigualdad social, aunque con un sistema electoral competitivo, generará desconfianza en las instituciones políticas, donde se agregaría a la desigualdad social los problemas de inseguridad y corrupción que presenta México antes y durante el proceso electoral 2018. Por lo que las instituciones no cumplen con lo que se considera por los ciudadanos su deber ser en relación con la generación de bienestar que le compete al estado.

En correspondencia con lo anterior se puede indicar que la desigualdad social en México se encuentra en niveles muy altos, ya que de acuerdo con el Consejo Nacional de Evaluación de la política de Desarrollo Social (CONEVAL, 2016) se reporta pobreza en el 43.6\% de la población. A su vez, con relación a la inseguridad de acuerdo con los indicadores que publica el Instituto Nacional de Estadística y Geografía (INEGI), al primer trimestre de 2019 el 74.6\% de la población mayor de 18 años percibe insegura en su ciudad.

En materia de corrupción, y de acuerdo con el índice de Percepción de la Corrupción 2018 de Transparencia Internacional, el estado mexicano presenta una calificación de 28 sobre 100 en el nivel de corrupción percibido dentro del sector público, que representan seis puntos menos en comparación con hace seis años. En lo que corresponde al nivel explicativo del consumo de contenido político online en relación con la confianza en las instituciones políticas y de gobierno, cabe mencionar no es muy relevante. Por ello, se podría solamente especular que posiblemente la atención online a candidatos y partidos políticos en campaña electoral podría generar expectativas de cambio en relación con los problemas presentados, que repercutirían en la confianza que se tiene frente algunas instituciones políticas y de gobierno.

Es importante destacar que en próximas investigaciones se deberá buscar determinar si el uso de internet con fines políticos influye en la percepción que tiene el ciudadano sobre problemas como la desigualdad social, la inseguridad y la corrupción y sí estos factores inciden en la confianza en las instituciones políticas y de gobierno.

\section{Declaración de conflicto de intereses}

Los autores no informaron ningún posible conflicto de intereses. 


\section{Fondos}

Este artículo se desprende del proyecto de investigación "La Cultura política en el escenario preelectoral 2018. Zona Metropolitana de Monterrey" financiado por la Facultad de Ciencias Políticas y Relaciones Internacionales de la Universidad Autonóma de Nuevo León.

\section{Referencias}

Amado, A., \& Tarullo, R. (2015). Las redes sociales en la comunicación política: ¿comunicación unidireccional o conversacional? Contratexto, (24), 97-111. doi: 10.26439/contratexto2015.no24.589

Asociación de Internet.mx. (2017). Hábitos de los Usuarios de Internet en México 2017. Ciudad de México, México: Autor. Recuperado de https://www.asociaciondeinternet.mx/es/component/remository/Habitos-de-Internet/13-Estudio-sobre-los-Habitos-de-

Bobbio, N., Matteucci, N., \& Pasquino, G. (2007). Diccionario de Política. Madrid, España: siglo XXI editores.

Borge, R., Cardenal, A. S., \& Malpica, C. (2012). El Impacto de Internet en la participación política: Revisando el papel del interés político. RBOR Ciencia, Pensamiento y Cultura, 188 (756), 733-750. doi: 10.3989/arbor.2012.756n4008

Boulianne, S. (2009). Does internet use affect engagement? A meta- analysis of research. Political Communication, 26 (2), 193-211. doi:10.1080/10584600902854363

Brussino, S., Rabbia, H. H., \& Sorribas, P. (2009). Perfiles socio-cognitivos de la participación política de los jóvenes. Interamerican Journal of Psychology, 43(2), 279-287.

Campos Freire, F. (2008). Las redes sociales trastocan los modelos de los medios de comunicación tradicionales. Revista Latina de Comunicación Social, 11(63), 287-293.

Consejo Nacional de Evaluación de la política de Desarrollo Social (2016). Medición de la pobreza en México. Recuperado de https://www.coneval.org.mx/Medicion/MP/Paginas/Pobreza 2016.aspx

Ceron, A. (2015). Internet, news, and political trust: The difference between social media and online media outlets. Journal of Computer-Mediated Communication. 20, 487-503. doi: $10.1111 /$ jec4.12129

Córdula Almeida, V., Barboza de Sousa Piantavinha, T., \& Bispo Dos Santos, V. (2015). Internet y redes sociales: ¿artefacto tecnológico o locus digital para la movilización política? Chasqui. Revista Latinoamericana de Comunicación, (128), 85-99.

Corduneanu, V. I., \& Muñiz, C. (2011). ¿Autoritarismo superado? Medios y actitudes políticas en el contexto mexicano. En C. Muñiz (Coord.), Comunicación, política y ciudadanía. Aportaciones actuales al estudio de la comunicación política (pp. 283-307). Ciudad de México, México: Fontamara.

Delli Carpini, M. (2000). Gen.com: Youth, civic engagement and the new information enviroment. Political Comunication, 17(4), 341-349. doi:10.1080/10584600050178942 
Díaz Jiménez, O. F., \& Muñiz, C. (2017). Los efectos de la comunicación política en el compromiso político de los jóvenes en la elección presidencial mexicana de 2012. Revista Mexicana de Ciencias Políticas y Sociales, 62(229), 181-221. doi:10.1016/So185-1918(17)30008-9

Durand Ponte, V. M. (1995). La cultura política autoritaria en México. Revista Mexicana de Sociología, 57(3), 67-103. doi:10.2307/3540863

ENCUP. (2012). Resultados de la Encuesta Nacional sobre Cultura Política y Prácticas Ciudadanas. Ciudad de México, México: Secretaría de Gobernación. Recuperado de http://bit.ly/UmmxZ5

ENDUTIH. (2017). Encuesta Nacional sobre Disponibilidad y Uso de Tecnologías de la Información en los Hogares. (Ciudad de México, México: INEGI. Recuperado de https://datos.gob.mx/busca/dataset/encuesta-nacional-sobre-disponibilidad-y-uso-de-tic-en-hogares-endutih

Galais, C. (2012). ¿Cada vez más apáticos? El desinterés político juvenil en España en perspectiva comparada. Revista Internacional de Sociología, 7o(1), 107-127. doi:10.3989/ris.2011.05.07

García Luengo, O., \& Maurer, M. (2009). A virtuous circle for all? Media exposure and political trust in Europe. CONfines, 5(9), 39-48.

Gershtenson, J., Ladewig, J., \& Plane, D. L. (2013). Political trust and the health of democracy in the United States. Rethinking traditional measures and interpretations. Revista Mexicana de Análisis Político y Administración Pública, 2(1), 111-129.

Gómez-Tagle, S., Tejera Gaona, H, Aguilar López, J., Ramírez, J., \& Díaz Jiménez, O. F. (2012). Informe de la Encuesta Nacional de Cultura Política de los Jóvenes. Ciudad de México, México: El Colegio de México. Recuperado de https://bit.ly/2pVyMw6

González de la Vega, A., Quintanilla, A., \& Tajonar M. (2010). Confianza en las instituciones políticas mexicanas: ¿capital social, valores culturales o desempeño? En A. Moreno (Ed.), La confianza en las instituciones. México en perspectiva comparada (pp. 63-99). Ciudad de México, México: Centro de Estudios Sociales y de Opinión Pública.

Kenski, K., \& Jominie, N. (2006). Connections between internet use and political efficacy, knowledge, and participation. Journal of Broadcasting \& Electronic Media, 5o(2) pp. 173-192. doi: 10.1207/s15506878jobem5002_1

Latinobarómetro. (2015). La confianza en América Latina 1995 - 2015. 20 años de opinión pública Latinoamérica. Recuperado de http://www.latinobarometro.org/LATDocs/Fo0005085-INFORME LB LA CONFIANZA 1995 2015.pdf

Lobera, J., \& Rubio, R. (2015). Nativos digitales: ¿̇hacia una nueva participación política? Revista de Estudios de Juventud, 108, 145-160.

Martínez, M.L., Silva, C., \& Hernández, A.C. (2010). ¿En qué Ciudadanía Creen los Jóvenes? Creencias, Aspiraciones de Ciudadanía y Motivaciones Para la Participación Sociopolítica. PSYKHE 19(2), 25-37. doi: 10.4067/So718-22282010000200004

Mazzoleni, G. (2010). La Comunicación Política. Madrid, España: Alianza Editorial.

Meneses, M. E., \& Bañuelos Capistrán, J. (2009). Internet y campañas electorales en México oportunidad postergada. [Serie Brevarios de Cultura Política Democrática No 8]. Ciudad de México, México: Instituto Electoral del Estado de México.

Moreno, A. (2009), La decisión electoral: Votantes, partidos y democracia en México. Ciudad de México, México: Miguel Ángel Porrúa. 
Moreno, A. (2010). Introducción. En Centro de Estudios Sociales y de Opinión Pública de la Cámara de Diputados (Ed.), La confianza en las instituciones. México en perspectiva comparada (pp. 11-59). Ciudad de México, México: CESOP.

Moya Sánchez, M., \& Herrera Damas, S. (2015a). Cómo puede contribuir Twitter a una comunicación política más avanzada. Arbor, 191 (774), 1-14. doi: 10.3989/arbor.2015.774n4012

Moya Sánchez, M., \& Herrera Damas, S. (2015b). Hacia una comunicación política avanzada en la Internet 2.0. Observatorio (OBS*) Journal, 9(4), 113-139. doi:10.15847/obsOBS942015907

Muñiz, C., \& Maldonado, L. (2011). Entre la movilización y el malestar mediático. Impacto de las prácticas comunicativas en las actitudes políticas de los jóvenes. Perspectivas de la Comunicación, 4(2), 32-54.

Norris, P. (2001). ¿Un círculo virtuoso? El impacto de las comunicaciones políticas en las democracias post-industriales. Revista Española de Ciencia Política, (4), 7-33.

Pérez, J. A., Peña, S., Genaut, A., Iturregui, L., \& Mendiguren, T. (2013). Comunicación política e Internet: Estrategias online de los partidos políticos vascos en las elecciones autonómicas de 2009. Mediatika, (14), 125-150.

Pew Research Center U.S. Politics and Policy. (2019). Public Trust in Government: 1958-2019. Recuperado de https://www.people-press.org/2019/04/11/public-trust-in-government-1958$\underline{2019 /}$

Putnam, R. D. (1995). Bowling Alone: America's Declining Social Capital. Journal of Democracy, 6(1), 65-78. doi: 10.1353/jod.1995.0002

Putnam, R. D. (2000). Bowling Alone: The Collapse and Revival of American Community. Nueva York, Estados Unidos: Simon \& Schuster.

Reyes Montes, M. C. (2007). Comunicación política y medios en México: el caso de la reforma a la Ley Federal de Radio y televisión. Convergencia. Revista de Ciencias Sociales, (43), 105-136.

Rivera, S. (2019). Confianza y participación política en América Latina. Revista Mexicana de Ciencias Políticas y Sociales, 64(235), 555-584. doi: 10.22201/fcpys.2448492xe.2019.235.65728

Saldierna, A. R., Marañon, F. J.; \& Muñiz, C. (2015). El papel de la comunicación en la generación de interés y conocimiento político entre los preciudadanos: aplicación del modelo O-S-R-O-R al caso mexicano. Revista Mexicana de Opinión Pública, (19), 147-165. doi: 10.1016/j.rmop.2015.03.003

Segovia, C., Haye, A., González, R., Manzi, J., \& Carvacho, H. (2008). Confianza en instituciones políticas en Chile: un modelo de los componentes centrales de juicios de confianza. Revista de ciencia política, 28(2), 39-60. doi: 10.4067/So718-090X2008000200002

Shah, D. V., Cho, J., Nah, S., Gotlieb, M. R., Hwang, H., Leem, N., Scholl, R. M., \& McLeod, D. M. (2007). Campaign ads, online messaging, and participation: Extending the communication mediation model. Journal of Communication, 57(4), 676-703. doi: 10.1111/j.14602466.2007.00363.x

Verge Mestre, T., \& Tormos Marín, R. (2012). La persistencia de las diferencias de género en el interés por la política. Reis, (138), 89-108. doi:10.5477/cis/reis.138.89

Yanes Mesa, R. (2007). La comunicación política y los nuevos medios de comunicación. Ámbitos, (16), 355-366. doi: 10.12795/Ambitos.2007.116.20 